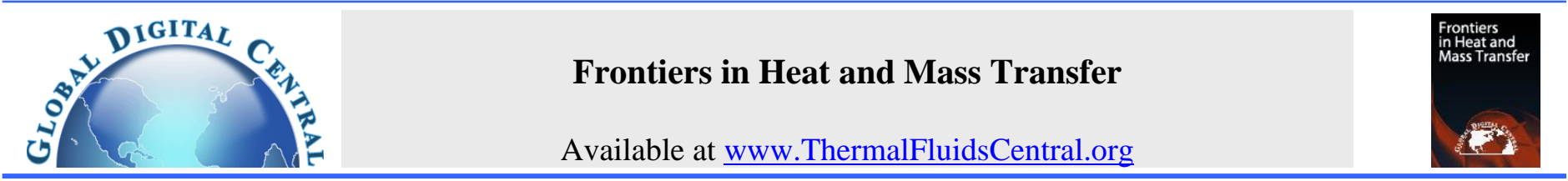

\title{
THERMODYNAMIC ANALYSIS FOR THE MHD FLOW OF TWO IMMISCIBLE MICROPOLAR FLUIDS BETWEEN TWO PARALLEL PLATES
}

\author{
J. Srinivas* and J. V. Ramana Murthy \\ Department of Mathematics, NIT Warangal 506004, Telangana State, India.
}

\begin{abstract}
The paper aims the heat transfer analysis for the flow of two immiscible micropolar fluids inside a horizontal channel, by the first and second laws of thermodynamics under the action of an imposed transverse magnetic field. The plates of the channel are maintained at constant temperatures higher than that of the fluid. The flow region consists of two zones, the flow of the heavier fluid taking place in the lower zone. The condition of hyper-stick is taken on the plates and continuity of velocity, micro-rotation, temperature, heat flux, shear stress and couple stress are imposed at the interface. The velocity, micro-rotation and temperature profiles are derived analytically and these are used to compute the dimensionless expressions for the entropy generation number and Bejan number. The results are presented graphically. It is observed that the imposed magnetic field reduces the entropy production rate near the walls.
\end{abstract}

Keywords: MHD flow, Immiscible fluids, Micropolar fluids, Entropy generation number, Bejan number.

\section{INTRODUCTION}

The optimal design of thermal systems can be achieved by minimizing the entropy generation rate in the systems. This issue has been the topic of great importance in many engineering fields such as heat exchangers, thermal insulation, petroleum and geothermal reservoirs, cooling of nuclear reactors, MHD power generators, cooling of electronic devices etc. Two decades earlier, measuring the entropy generation rate of such complex systems which have different geometries in the presence and absence of a magnetic field in a porous or non-porous channel was restricted to the first law of thermodynamics. In recent days, to estimate the entropy generation rate it is observed that use of the second law of thermodynamics, is more reliable than the calculations based on first law. In almost all thermal systems, based on the second law, the efficiency of the system can be defined in terms of the ratio of actual thermal efficiency to reversible thermal efficiency under the same conditions. In order to enhance the thermal performance of a system, i.e., to reduce the destruction of the available work, the entropy generation rate must be minimized (Bejan, 1980). The entropy generation rate is associated with thermodynamic irreversibility, which is common in all types of heat transfer processes. Different sources like heat transfer across finite temperature gradient, characteristic of convective heat transfer, fluid friction, viscosity effects within the fluid and at the solid boundaries, chemical reactions, body force effect, coupling effects between heat and mass transfer, viscous dissipation effect etc. are all responsible for entropy generation rate. Bejan (1982, 1979) discussed different reasons behind the entropy generation rate in many thermal systems. Generation of entropy destroys available work of a system. Therefore, it makes a good engineering sense to focus on the irreversibility of heat transfer and fluid flow processes and try to understand the mechanism of entropy generation rate. Also he performed an analytical study to show how the entropy generation rate can be calculated in fundamental convective heat transfer problems such as pipe flow, boundary layer flow over a flat plate, single cylinder in cross flow and flow in the entrance region of a flat rectangular duct. He demonstrated how the flow geometric parameters may be selected in order to minimize the irreversibility associated with a specific convective heat transfer.

There are many problems in the fields of hydrology and reservoir mechanics in which systems involving two or more immiscible fluids of different densities/viscosities flowing in same pipe or channel or through porous media are encountered. Typical fluid examples of these systems are: air-water, water-salt water, oil-water, gas-oil, and gas-oil-water systems. These are referred to as multi-phase flows in literature. Blood flow in arteries has been studied by many researchers considering the flow of blood as a two phase flow. Several investigations of multi-phase flows are reported by various researchers (e.g., Bird et al., 1960; Kapur, 1964; Bhattacharya, 1968; Chaturani and Samy, 1985; Rao and Usha, 1979; Bakhtiyarov and Siginer, 1997). The flow and heat transfer in immiscible fluids are of special importance in the petroleum extraction and transport problem. Heat transfer in immiscible flows was discussed by many authors (e.g., Chamkha, 2000; Umavathi et al., 2005; Malashetty et al., 2006; Muthuraj and Srinivas, 2010; Prathap Kumar et al., 2011).

In the last few decades, a lot of study has been developed in order to investigate the effect of a magnetic field on different geometries

${ }^{\dagger}$ Corresponding author. Email:j.srinivasnit@gmail.com 
(Chamkha, 1998; Chamkha and Quadri, 2001). Alpher (1961) discussed the heat transfer in magnetohydrodynamic flow between parallel plates. Nikodijevic et al. (2011) studied the MHD Couette two-fluid flow and heat transfer in the presence of a uniform inclined magnetic field. It is also to be noted that the analytical study of first and second laws of thermodynamics for the flow within the channel in the presence of a magnetic field is significant in many industrial applications, such as MHD marine propulsion, MHD generators, pumps, accelerators, flow meters, filtration, geothermal systems and has applications in nuclear reactors too. Several works have been reported on the effect of MHD flow on the entropy generation for various flows and geometries (e.g., Damseh et al., 2008; Mahian et al., 2012; Kiyasatfar et al., 2012; Komurgoz et al., 2012). In recent years, the fluid flow and entropy generation in two immiscible fluids in a channel have received considerable attention of researchers. Kamisli and Hakan (2008) considered the fluid flow and entropy generation in two immiscible fluids in a channel. These authors explained very nicely the thermodynamic interface conditions involved in a flow of immiscible fluids and made a significant observation that minimum temperature gradient in the transverse direction of the flow offers minimum entropy generation near the plates.

The theory of a micropolar fluid was initiated by Eringen (1966, 1960 ) in 1966. This theory accounts for the internal characteristics of the substructure particles with the assumption that they are allowed to undergo a rotation independent of their linear velocity. Micropolar fluids represent fluids consisting of rigid randomly oriented particles suspended in a viscous medium when the deformation of the particles is ignored. The equations of motion characterizing a micropolar fluid flow are nonlinear in nature (as in the case of Newtonian viscous fluids) and are constituted by a coupled system of vector differential equations in velocity $\bar{q}$ and micro-rotation $\bar{\nu}$ (which is independent of $\bar{q}$ ). To understand the departure from the viscous fluid flow model, several problems that were studied in viscous fluid flow theory, have also been studied in the realm of micropolar fluids. This theory can be used to explain the flow of colloidal fluids, liquid crystals, animal blood etc. An account of the early developments in polar fluid theory can be found in the book by Stokes (1984). Ariman and Cakmak (1968) discussed some basic viscous flows in micropolar fluids. A detailed survey of microcontinuum fluid mechanics with several applications in physiological fluid flows has been presented by Ariman et al. (1973). Interesting aspects of the theory and applications of micropolar fluids are dealt in the books by Eringen (1960) and Lukaszewicz (1999).

Thermodynamic analysis in the case of viscous fluids was carried out by many researchers (e.g., Kiyasatfar et al., 2012; Kamisli and Hakan, 2008). But very less attention has been paid in this direction for the micropolar fluid flows. Since micropolar fluids are known to have rotational effects, these fluids may help to increase the exergy. Hence, the present study aims to study a comparatively involved problem dealing with the entropy generation characteristics in a channel of two immiscible micropolar fluid flows under the influence of a transverse magnetic field.

\section{MATHEMATICAL FORMULATION}

Consider the flow of two immiscible micropolar fluids between two parallel plates extending in the axial direction and distant $2 h$ apart. $X$ and $Y$ are the axial and transverse coordinates respectively with the origin at the center of the channel (Fig. 2). The length of the plates is much greater than the distance between them so that the flow at any point in the $\mathrm{X}$-direction is same. Fluid flow is generated due to a constant pressure gradient which acts at the mouth of the channel. A constant transverse magnetic field is applied on the plates. The fluid in the lower zone (viscosity $\mu_{1}$, micropolarity $\kappa_{1}$, density $\rho_{1}$ and thermal conductivity $k_{1}$ ) occupies the region (i.e. $-h \leq Y \leq 0$ ) comprising the lower half of the channel and this region is named zone I. The fluid in the upper zone (viscosity $\mu_{2}$, micropolarity $\kappa_{2}$, density $\rho_{2}\left(<\rho_{1}\right)$ and thermal conductivity $k_{2}$ ) is assumed to occupy the upper half of the channel (i.e. $0 \leq Y \leq h$ ), and this region is called zone II. The equations for the flow and energy in zone I and II (i.e. $-h \leq Y \leq h$ ) are assumed to be governed by micropolar fluid flow equations as given by Eringen (1966).

$$
\begin{gathered}
\frac{\partial \rho}{\partial t}+\nabla \cdot(\rho \overrightarrow{\mathbf{q}})=0 \\
\rho \frac{d \overrightarrow{\mathbf{q}}}{d t}=\rho \overrightarrow{\mathbf{f}}-\nabla P+\kappa \nabla \times \overrightarrow{\boldsymbol{\nu}}-(\mu+\kappa) \nabla \times \nabla \times \overrightarrow{\boldsymbol{q}}+(\lambda+2 \mu+\kappa) \nabla(\nabla \cdot \overrightarrow{\boldsymbol{q}})+\overrightarrow{\boldsymbol{J}} \times \overrightarrow{\boldsymbol{H}} \\
\rho j \frac{d \overrightarrow{\boldsymbol{\nu}}}{d t}=\rho \overrightarrow{\boldsymbol{\ell}}-2 \kappa \overrightarrow{\boldsymbol{\nu}}+\kappa \nabla \times \overrightarrow{\boldsymbol{q}}-\gamma \nabla \times \nabla \times \overrightarrow{\boldsymbol{\nu}}+(\alpha+\beta+\gamma) \nabla(\nabla \cdot \overrightarrow{\boldsymbol{\nu}}) \\
\rho \frac{d E}{d t}=-P(\nabla)
\end{gathered}
$$

where

$$
\begin{aligned}
\rho \Phi=\lambda(\nabla \cdot \overrightarrow{\boldsymbol{q}})^{2} & +2 \mu(D: D)+4 \kappa\left(\frac{1}{2} \nabla \times \overrightarrow{\boldsymbol{q}}-\overrightarrow{\boldsymbol{\nu}}\right)^{2} \\
& +\alpha(\nabla \cdot \overrightarrow{\boldsymbol{\nu}})^{2}+\gamma(\nabla \overrightarrow{\boldsymbol{\nu}}: \nabla \overrightarrow{\boldsymbol{\nu}})+\beta\left(\nabla \overrightarrow{\boldsymbol{\nu}}:(\nabla \overrightarrow{\boldsymbol{\nu}})^{T}\right)
\end{aligned}
$$

The equations (1)-(4) represent conservation of mass, balance of linear momentum, micro-rotation and energy equation respectively. The scalar quantities $\rho$ and $j$ are respectively the density and gyration coefficient and are assumed to be constants. The vectors $\vec{q}, \vec{\nu}, \vec{f}$ and $\vec{\ell}$ are the velocity, micro-rotation, body force per unit mass and body couple per unit mass respectively. $P$ is the fluid pressure at any point. $\overrightarrow{\boldsymbol{J}}$ is the current density and $\overrightarrow{\boldsymbol{H}}$ is the total magnetic field $\left(\overrightarrow{\boldsymbol{H}}=\overrightarrow{\boldsymbol{H}}_{o}+\overrightarrow{\boldsymbol{h}}_{o}\right)$ where $\overrightarrow{\boldsymbol{H}}_{o}$ is the applied magnetic field and $h_{o}$ is the induced magnetic field $\left(h_{o}<<H_{o}\right)$. The material constants $(\lambda, \mu, \kappa)$ are viscosity coefficients and $(\alpha, \beta, \gamma)$ are gyro-viscosity coefficients. In the energy equation, $\Phi$ is the dissipation function of mechanical energy per unit mass, $D$ denotes the deformation tensor, $E$ is the specific internal energy and $\overrightarrow{\mathbf{h}}$ is the heat flux, where $k$ is the thermal conductivity.

The current density $\overrightarrow{\boldsymbol{J}}$, magnetic field $\overrightarrow{\boldsymbol{H}}$ and electric field $\overrightarrow{\boldsymbol{E}}$ are related by Maxwell's equations $\nabla \times \overrightarrow{\boldsymbol{E}}=0, \nabla \cdot \overrightarrow{\boldsymbol{H}}=0, \nabla \times \overrightarrow{\boldsymbol{H}}=\mu^{1} \overrightarrow{\boldsymbol{J}}$, $\nabla \cdot \overrightarrow{\boldsymbol{J}}=0, \overrightarrow{\boldsymbol{J}}=\sigma(\overrightarrow{\boldsymbol{E}}+\overrightarrow{\boldsymbol{q}} \times \overrightarrow{\boldsymbol{H}})$ where $\mu^{1}$ is the magnetic permeability and $\sigma$ is the electric conductivity.

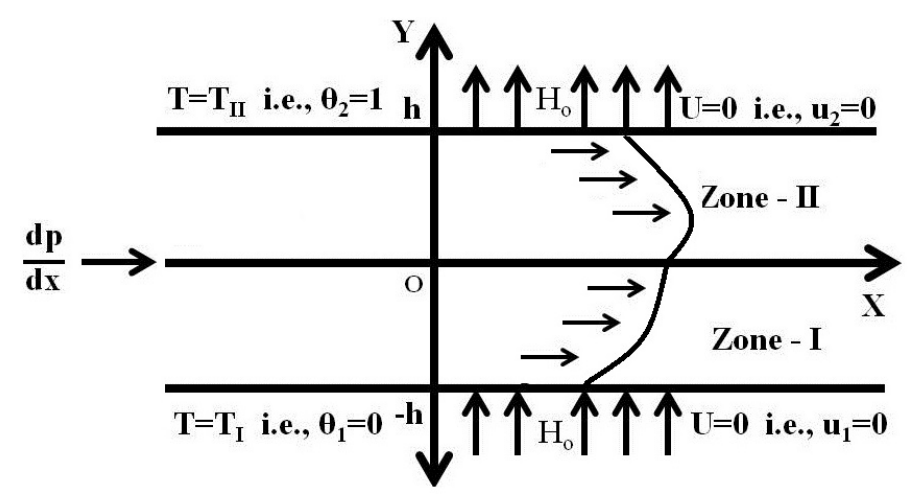

Fig. 1 Geometry of the problem

To develop the governing equations for the problem considered, the following assumptions are made:

1. The flow is assumed to be one-dimensional, steady, laminar, and incompressible.

2. The gravity effect is negligible and the Lorentz force $(\overrightarrow{\boldsymbol{J}} \times \overrightarrow{\boldsymbol{H}})$ is the only body force acting on the fluid. 
3. The magnetic Reynolds number is assumed to be small, so that the induced magnetic field is neglected and the Hall-effect of magnetohydrodynamics is assumed to be negligible.

We assume that the velocity of the fluid is $\overrightarrow{\boldsymbol{q}}=(U(Y), 0,0)$ and microrotation vector as $\overrightarrow{\boldsymbol{\nu}}=(0,0, C(Y))$. The flow is subjected to a uniform applied magnetic field $H_{o}$ in the positive y-direction.i.e., $\overrightarrow{\boldsymbol{H}}=H_{o} \overrightarrow{\boldsymbol{j}}$ which gives $\overrightarrow{\boldsymbol{J}} \times \overrightarrow{\boldsymbol{H}}=-\sigma H_{o}^{2} \overrightarrow{\boldsymbol{q}}$.

Introducing the non-dimensional variables: $x=\frac{X}{h}, y=\frac{Y}{h}, u=\frac{U}{U_{o}}$, $p=\frac{P}{\rho_{1} U_{o}^{2}}, C=\frac{\mathcal{C} U_{o}}{h}$ where $U_{o}$ is the maximum velocity of the fluid in the channel, we get the following sets of non-dimensional form of governing equations (neglecting body couples) and boundary conditions corresponding to the flow in the two zones.

\section{GOVERNING EQUATIONS}

Zone I: $(-1 \leq y \leq 0)$

The governing equations in zone I are:

$$
\begin{aligned}
\frac{d^{2} u_{1}}{d y^{2}}+c_{1} \frac{d \mathcal{C}_{1}}{d y}-H a^{2}\left(1-c_{1}\right) u_{1} & =\left(1-c_{1}\right) R e \frac{d p}{d x} \\
\frac{d^{2} \mathcal{C}_{1}}{d y^{2}}-s_{1} \frac{d u_{1}}{d y}-2 s_{1} \mathcal{C}_{1} & =0
\end{aligned}
$$

Zone II: $(0 \leq y \leq 1)$

The governing equations in zone II are:

$$
\begin{gathered}
\frac{d^{2} u_{2}}{d y^{2}}+c_{2} \frac{d \mathcal{C}_{2}}{d y}-\frac{n_{\sigma}}{n_{\mu}} H a^{2}\left(1-c_{2}\right) u_{2}=\frac{1}{n_{\mu}}\left(1-c_{2}\right) \operatorname{Re} \frac{d p}{d x} \\
\frac{d^{2} \mathcal{C}_{2}}{d y^{2}}-s_{2} \frac{d u_{2}}{d y}-2 s_{2} \mathcal{C}_{2}=0
\end{gathered}
$$

where $R e=\frac{\rho_{1} U_{o} h}{\mu_{1}}$ is the Reynolds number, $H a^{2}=\frac{\sigma_{1} H_{o}^{2} h^{2}}{\mu_{1}}$ is the Hartmann number, $n_{\mu}=\frac{\mu_{2}}{\mu_{1}}$ is the viscosity ratio, $n_{\sigma}=\frac{\sigma_{2}}{\sigma_{1}}$ is the electric conductivity ratio, $c_{i}=\frac{\kappa_{i}}{\mu_{i}+\kappa_{i}}$ is the micropolarity parameter and $s_{i}=\frac{\kappa_{i} h^{2}}{\gamma_{i}}$ is the couple stress parameter $(\mathrm{i}=1,2)$.

\section{BOUNDARY AND INTERFACE CONDITIONS}

A characteristic feature of the two-layer flow problem is the coupling across liquid-liquid interfaces. The liquid layers are mechanically coupled via transfer of momentum across the interfaces. Transfer of momentum results from the continuity of tangential velocity and a stress balance across the interface.

To determine the velocity and micro-rotation components $u_{1}(y)$, $\mathcal{C}_{1}(y), u_{2}(y)$ and $\mathcal{C}_{2}(y)$ in the zones I and II described above, we adopt the following boundary and interface conditions:

Zone $\mathrm{I}$ is constituted by the fixed lower plate given by $y=-1$ and a fluid-fluid interface defined by $y=0$. Zone II is constituted by the fluid interface given by $y=0$ and the fixed upper plate given by $y=1$.

In view of the no-slip condition (Sandeep and Deshpande (2003)) on the static boundaries, we have to prescribe velocity as:

$$
u_{1}(y)=0 \text { on } y=-1 \text { and } u_{2}(y)=0 \text { on } y=1
$$

which represent the no-slip condition.

The microrotation vector on the boundary $=$ angular velocity of the fluid on the boundary. i.e., $C_{\text {wall }}=\frac{1}{2}\left(\nabla \times \overrightarrow{\boldsymbol{q}}_{\text {wall }}\right)$. A more general condition is taken as $\mathcal{C}_{\text {wall }}=\frac{n}{2}\left(\nabla \times \overrightarrow{\boldsymbol{q}}_{\text {wall }}\right)$ where $0 \leq n \leq 1$ (Refer Lukaszewicz (1999), page 31). This value of $n$ indicates the concentration of micropolarity or interaction of fluid particles with the boundary. The case $n=0$ indicates $\mathcal{C}=0$ at the plates. It represents the flow of concentrated particles in which the microelements close to the wall surface are unable to rotate (Jena and Mathur, 1981). While there has been theoretical justification for no-slip condition for the velocity on the boundary, the boundary condition for the micro-rotation vector $\overrightarrow{\boldsymbol{\nu}}$ needs to be established in a rigorous form. The hyper-stick condition on the boundary as given in Lukaszewicz (1999) is the one adopted here following the studies made by Rees and Bossom (1996); Bhattacharyya et al. (2012) and several workers in micropolar fluid flow and hence we take that the microrotation vanishes on the static boundaries.

$$
\mathcal{C}_{1}(y)=0 \text { on } y=-1 \text { and } \mathcal{C}_{2}(y)=0 \text { on } y=1
$$

At the fluid-fluid interface $y=0$, we assume that the velocity, microrotation, shear stress and couple stress components are continuous. This implies

$$
\begin{array}{r}
u_{1_{\left(0^{-}\right)}}=u_{2\left(0^{+}\right)}, \mathcal{C}_{1\left(0^{-}\right)}=\mathcal{C}_{2\left(0^{+}\right)},\left.\tau_{1 x y}\right|_{\left(0^{-}\right)}=\left.\tau_{2 x y}\right|_{\left(0^{+}\right)} \text {and } \\
\left.M_{1 x y}\right|_{\left(0^{-}\right)}=\left.n_{\beta} M_{2 x y}\right|_{\left(0^{+}\right)}
\end{array}
$$

The last two conditions of (11) give us

$$
\begin{gathered}
\left.\tau_{1 x y}\right|_{\left(0^{-}\right)}=\left.\tau_{2 x y}\right|_{\left(0^{+}\right)} \Rightarrow\left[\left.\frac{\partial u_{1}}{\partial y}\right|_{\left(0^{-}\right)}+\left.2 c_{1} \mathcal{C}_{1}\right|_{\left(0^{-}\right)}\right] \\
=n_{\mu}\left(\frac{1-c_{1}}{1-c_{2}}\right)\left[\left.\frac{\partial u_{2}}{\partial y}\right|_{\left(0^{+}\right)}+\left.2 c_{2} \mathcal{C}_{2}\right|_{\left(0^{+}\right)}\right] \\
\left.M_{1 x y}\right|_{\left(0^{-}\right)}=\left.\left.M_{2 x y}\right|_{\left(0^{+}\right)} \Rightarrow \frac{\partial \mathcal{C}_{1}}{\partial y}\right|_{\left(0^{-}\right)}=\left.n_{\beta} \frac{\partial \mathcal{C}_{2}}{\partial y}\right|_{\left(0^{-}\right)} .
\end{gathered}
$$

At the interface as there is no mass transfer, it is justified to assume that there is no velocity in normal direction and that the tangential velocity and microrotation are continuous. Also interface is assumed to be a smooth surface representing force balance and hence shear stress and couple stress are prescribed to be continuous.

\section{SOLUTION OF THE PROBLEM}

\subsection{Velocity and Microrotation distributions}

Solution of momentum equations subject to the conditions through (9)(11) are obtained as:

Zone I: $(-1 \leq y \leq 0)$

Eliminating $\mathcal{C}_{1}$ from (5), (6) we have

$$
\begin{array}{r}
\frac{d^{4} u_{1}}{d y^{4}}-\left(H a^{2}\left(1-c_{1}\right)+s_{1}\left(2-c_{1}\right)\right) \frac{d^{2} u_{1}}{d y^{2}}+2 s_{1}\left(1-c_{1}\right) u_{1} \\
=-2 s_{1}\left(1-c_{1}\right) \operatorname{ReB}
\end{array}
$$

substituting $\frac{d \mathcal{C}_{1}}{d y}$ from (5) in (6) we have

$$
\mathcal{C}_{1}(y)=-\frac{1}{2 c_{1} s_{1}}\left[\left(H a^{2}\left(1-c_{1}\right)-c_{1} s_{1}\right) \frac{d u_{1}}{d y}-\frac{d^{3} u_{1}}{d y^{3}}\right]
$$

solving (13) we get,

$$
\begin{array}{r}
u_{1}(y)=c_{11} \cosh \alpha_{1} y+c_{12} \sinh \alpha_{1} y+c_{13} \cosh \alpha_{2} y+c_{14} \sinh \alpha_{2} y \\
+\frac{1}{H a^{2}} \operatorname{ReB}
\end{array}
$$

and substituting $u_{1}(y)$ in (14) we have

$$
\begin{gathered}
\mathcal{C}_{1}(y)=\frac{1}{2 c_{1} s_{1}}\left[\alpha_{1}\left(H a^{2}\left(1-c_{1}\right)-c_{1} s_{1}-\alpha_{1}^{2}\right)\right. \\
\left(c_{11} \sinh \alpha_{1} y+c_{12} \cosh \alpha_{1} y\right)+\alpha_{2}\left(H a^{2}\left(1-c_{1}\right)-c_{1} s_{1}-\alpha_{2}^{2}\right) \\
\left.\left(c_{13} \sinh \alpha_{2} y+c_{14} \cosh \alpha_{2} y\right)\right]
\end{gathered}
$$


Zone II: $(0 \leq y \leq 1)$

Eliminating $\mathcal{C}_{2}$ from (7), (8) we have

$$
\begin{aligned}
\frac{d^{4} u_{2}}{d y^{4}}-\left(H a^{2} \frac{n_{\sigma}}{n_{\mu}}\left(1-c_{2}\right)+s_{2}(2-\right. & \left.\left.c_{2}\right)\right) \frac{d^{2} u_{2}}{d y^{2}}+2 s_{2}\left(1-c_{2}\right) u_{2} \\
& =-2 s_{2}\left(1-c_{2}\right) \frac{1}{n_{\mu}} \operatorname{ReB}
\end{aligned}
$$

substituting $\frac{d \mathcal{C}_{2}}{d y}$ from (7) in (8) we have

$$
\left.\mathcal{C}_{2}(y)=-\frac{1}{2 c_{2} s_{2}}\left[\left(H a^{2} \frac{n_{\sigma}}{n_{\mu}}\left(1-c_{2}\right)-c_{2} s_{2}\right)\right) \frac{d u_{2}}{d y}-\frac{d^{3} u_{2}}{d y^{3}}\right]
$$

solving (17) we get,

$$
\begin{array}{r}
u_{2}(y)=c_{21} \cosh \alpha_{3} y+c_{22} \sinh \alpha_{3} y+c_{23} \cosh \alpha_{4} y+c_{24} \sinh \alpha_{4} y \\
-\frac{1}{n_{\mu}} \frac{1}{H a^{2}} \operatorname{ReB}
\end{array}
$$

and substituting $u_{2}(y)$ in (18), we have

$$
\begin{gathered}
\mathcal{C}_{2}(y)=\frac{1}{2 c_{2} s_{2}}\left[\alpha_{3}\left(H a^{2}\left(1-c_{2}\right) \frac{n_{\sigma}}{n_{\mu}}-c_{2} s_{2}-\alpha_{3}^{2}\right)\right. \\
\left(c_{21} \sinh \alpha_{3} y+c_{22} \cosh \alpha_{3} y\right)+\alpha_{4}\left(H a^{2}\left(1-c_{2}\right) \frac{n_{\sigma}}{n_{\mu}}-c_{2} s_{2}-\alpha_{4}^{2}\right) \\
\left.\left(c_{23} \sinh \alpha_{4} y+c_{24} \cosh \alpha_{4} y\right)\right]
\end{gathered}
$$

where $\alpha_{1}^{2}, \alpha_{2}^{2}$ are the roots of $x^{2}-a_{1} x+a_{2}=0$ and $\alpha_{3}^{2}, \alpha_{4}^{2}$ are the roots of $x^{2}-b_{1} x+b_{2}=0$ and $a_{1}=\alpha_{1}^{2}+\alpha_{2}^{2}=H a^{2}\left(1-c_{1}\right)+s_{1}\left(2-c_{1}\right), a_{2}=$ $\alpha_{1}^{2} \alpha_{2}^{2}=4 s_{1}\left(1-c_{1}\right) H a^{2}, b_{1}=\alpha_{3}^{2}+\alpha_{4}^{2}=\frac{n_{\sigma}}{n_{\mu}} H a^{2}\left(1-c_{2}\right)+s_{2}\left(2-c_{2}\right)$, $b_{2}=\alpha_{3}^{2} \alpha_{4}^{2}=4 \frac{n_{\sigma}}{n_{\mu}} s_{2}\left(1-c_{2}\right) H a^{2}$

The solutions $u_{1}(y), \mathcal{C}_{1}(y)$ and $u_{2}(y), \mathcal{C}_{2}(y)$ involve eight constants $c_{11}, c_{12}, c_{13}, c_{14}, c_{21}, c_{22}, c_{23}$ and $c_{24}$. These constants are found from the boundary conditions given in (9)-(11) and these are obtained using MATHEMATICA. As the expressions are cumbersome, they are not presented here.

\subsection{Temperature distributions}

In the present problem, it is assumed that the two walls are maintained at constant and different temperatures $T_{I}$ and $T_{I I} \quad\left(T_{I}<T_{I I}\right)$.

The governing equation for the temperature $T_{1}$ of the conducting fluid in zone $\mathrm{I}$ is then given by

$k_{1} \frac{d^{2} T_{1}}{d Y^{2}}=-\left[\mu_{1}\left(\frac{d U_{1}}{d Y}\right)^{2}+\kappa_{1}\left(\frac{d U_{1}}{d Y}+2 C_{1}\right)^{2}+\beta_{1}\left(\frac{d C_{1}}{d Y}\right)^{2}+\sigma_{1} H_{o}^{2} U_{1}^{2}\right]$

The governing equation for the temperature $T_{2}$ of the conducting fluid in zone II is then given by

$k_{2} \frac{d^{2} T_{2}}{d Y^{2}}=-\left[\mu_{2}\left(\frac{d U_{2}}{d Y}\right)^{2}+\kappa_{2}\left(\frac{d U_{2}}{d Y}+2 C_{2}\right)^{2}+\beta_{2}\left(\frac{d C_{2}}{d Y}\right)^{2}+\sigma_{2} H_{o}^{2} U_{2}^{2}\right]$

In order to non-dimensionalize the above equations, the following transformation is used in addition to those already introduced in above: $\theta=$ $\frac{T-T_{I}}{T_{I I}-T_{I}}$.

The Equations (21) and (22) are then reduced to the following form:

$$
\begin{array}{r}
\frac{d^{2} \theta_{1}}{d y^{2}}=-B r\left[\left(\frac{d u_{1}}{d y}\right)^{2}+\left(\frac{c_{1}}{1-c_{1}}\right)\left(\frac{d u_{1}}{d y}+2 \mathcal{C}_{1}\right)^{2}+\delta_{1}\left(\frac{d \mathcal{C}_{1}}{d y}\right)^{2}\right. \\
\left.+H a^{2} u_{1}^{2}\right]
\end{array}
$$

$$
\begin{array}{r}
\frac{d^{2} \theta_{2}}{d y^{2}}=-\frac{B r}{n_{k}}\left[n _ { \mu } \left(\left(\frac{d u_{2}}{d y}\right)^{2}\right.\right. \\
\left.+\left(\frac{c_{2}}{1-c_{2}}\right)\left(\frac{d u_{2}}{d y}+2 \mathcal{C}_{2}\right)^{2}\right) \\
\left.+n_{\beta} \delta_{1}\left(\frac{d \mathcal{C}_{2}}{d y}\right)^{2}+n_{\sigma} H a^{2} u_{2}^{2}\right]
\end{array}
$$

where $B r=E k \operatorname{Pr}$ is the Brinkman number, $E k=\frac{U_{o}^{2}}{c_{p_{1}}\left(T_{I I}-T_{I}\right)}$ is the Eckert number, $\operatorname{Pr}=\frac{\mu_{1} c_{p_{1}}}{k_{1}}$ is the Prandtl number, $n_{k}=\frac{k_{2}}{k_{1}}$ is the thermal conductivity ratio, $n_{\beta}=\frac{\beta_{2}}{\beta_{1}}$ is the couple stress co-efficient ratio and $\delta_{1}=\frac{\beta_{1}}{\mu_{1} h^{2}}$ is the couple stress parameter.

In the non-dimensional form, the boundary conditions for temperature are as below:

(i) at the lower and upper plate boundaries the temperatures are respectively,

$$
\theta_{1}=0 \text { at } y=-1 \text { and } \theta_{2}=1 \text { at } y=1
$$

(ii) at the fluid interface temperature $(\theta)$ and heat flux $(\overrightarrow{\mathbf{h}})$ are continuous:

$$
\theta_{1}=\theta_{2} \text { and } \frac{d \theta_{1}}{d y}=n_{k} \frac{d \theta_{2}}{d y} \text { at } y=0
$$

The solutions of Eqns. (23) and (24) with boundary and interface conditions are solved analytically and they are lengthy and are not shown here. The solution involves four constants and they are found from the four boundary conditions (Eqns. (25) and (26)) and are obtained using MATHEMATICA.

\subsection{Nusselt number}

The heat transfer coefficient at the walls is given by Fourier's law of heat conduction $\overrightarrow{\mathbf{h}}=-k \nabla T$. In non-dimensional form this represents Nusselt number $\left.N u=-\frac{d \theta}{d y}\right]_{y= \pm 1}$. The Nusselt number $\mathrm{Nu}$ is the ratio of convective to conductive heat transfer across (normal to) the wall.

\section{ENTROPY GENERATION ANALYSIS}

\subsection{The volumetric entropy generation}

If we take an infinitesimal fluid element in each zone and assume that the element as an open thermodynamic system subjected to mass fluxes, energy transfer and entropy transfer interactions through a fixed control surface, the volumetric rate of entropy generation for incompressible micropolar fluid is given as

$$
\begin{aligned}
\left(S_{i}\right)_{G}=\underbrace{\frac{k_{i}}{T_{o}^{2}}\left(\frac{\partial T_{i}}{\partial Y}\right)^{2}}_{\geq 0}+\underbrace{\frac{\mu_{i}}{T_{o}}\left(\frac{\partial U_{i}}{\partial Y}\right)^{2}}_{\geq 0}+\underbrace{\frac{\kappa_{i}}{T_{o}}\left(\frac{\partial U_{i}}{\partial Y}+2 C_{i}(Y)\right)^{2}}_{\geq 0} \\
+\underbrace{\frac{\beta_{i}}{T_{o}}\left(\frac{\partial C_{i}}{\partial Y}\right)^{2}}_{\geq 0}+\underbrace{\frac{1}{T_{o} \sigma_{i} H_{o}^{2} U_{i}^{2}}}_{\geq 0}
\end{aligned}
$$

where the value of i can be either 1 or 2 that represents zone I or zone II, respectively. Equation (27) clearly shows contributions of three sources of entropy generation rate. The first term on the right-hand side of Equation (27) is the entropy production due to heat transfer across a finite temperature difference, the second, third, and fourth terms are due to viscous dissipation and the fifth term is due to the effect of the magnetic field.

\subsection{The characteristic entropy generation rate}

The characteristic entropy generation rate $S_{G, C}$ is defined as,

$$
S_{G, C}=\left[\frac{\left(\overrightarrow{\mathbf{h}}_{1}\right)^{2}}{k_{1} T_{o}^{2}}\right]=\left[\frac{k_{1}(\Delta T)^{2}}{h^{2} T_{o}^{2}}\right]
$$


In the above equation, $\overrightarrow{\mathbf{h}}_{1}$ is the heat flux, $T_{o}$ is the average, characteristic, absolute reference temperature of the medium, $\Delta T=T_{I I}-T_{I}$ and $h$ is the half of transverse distance of the channel.

\subsection{The entropy generation number}

According to Bejan (1996), the dimensionless form of entropy generation is the entropy generation number $N s$ and which is, by definition, equal to the ratio of actual generation rate to a characteristic entropy transfer rate. The entropy generation number for each fluid is given by

$$
\begin{array}{r}
N s_{1}=\left(\frac{d \theta_{1}}{d y}\right)^{2}+\left(\frac{B r}{\Omega}\right)\left[\left(\frac{d u_{1}}{d y}\right)^{2}+\left(\frac{c_{1}}{1-c_{1}}\right)\left(\frac{d u_{1}}{d y}+2 \mathcal{C}_{1}\right)^{2}\right. \\
\left.+\delta_{1}\left(\frac{d \mathcal{C}_{1}}{d y}\right)^{2}+H a^{2} u_{1}^{2}\right] \\
N s_{2}=n_{k}\left(\frac{d \theta_{2}}{d y}\right)^{2}+\left(\frac{B r}{\Omega}\right)\left[n _ { \mu } \left(\left(\frac{d u_{2}}{d y}\right)^{2}+\right.\right. \\
\left.\left.\left(\frac{c_{2}}{1-c_{2}}\right)\left(\frac{d u_{2}}{d y}+2 \mathcal{C}_{2}\right)^{2}\right)+\delta_{1} n_{\beta}\left(\frac{d \mathcal{C}_{2}}{d y}\right)^{2}+\frac{n_{k} n_{\sigma}}{n_{\mu}} H a^{2} u_{2}^{2}\right]
\end{array}
$$

where $B r=\left(\frac{\mu_{1} U_{o}^{2}}{k_{1} \Delta T}\right)$ is the Brinkman number, which determines importance of viscous dissipation because of the fluid frictions relative to the conduction heat flow resulting from the impressed temperature difference and $\Omega=\left(\frac{\Delta T}{T_{o}}\right)$ is the temperature difference number. This number is a dimensionless parameter always present in the second law treatment of heat transfer processes. In most applications of engineering interest, we find $\Omega \leq 1$ (Bejan, 1979).

\subsection{The viscous dissipation parameter}

The viscous dissipation parameter is an important dimensionless number for the irreversibility analysis. It determines the relative importance of the viscous effects for the entropy generation and it is equal to the ratio of Brinkman number to the dimensionless temperature difference i.e., $(B r / \Omega)$. It is desirable to consider the $E k$ and $P r$ in a group called the Brinkman number $(B r=E k . P r)$ for evaluating the relative importance of the energy due to viscous dissipation to the energy due to heat conduction. It was reported that $\mathrm{Br}$ is much less than unity for many engineering processes (Bejan, 1979).

\subsection{The Bejan number}

The domination of the irreversibility mechanisms is physically important. The entropy generation originates from both heat transfer and viscous friction, these effects should be separately investigated to see the dominance of one over the other. The entropy generation number is unable to to give any idea about the relative importance of friction and heat transfer effects. An alternate parameter, called Bejan number $(B e)$, is introduced for this purpose and it is achieving an increasing popularity among researchers studying the second law. This parameter was defined by Paoletti et al. (1989) as ratio of irreversibility due to heat transfer to the total irreversibility (sum of heat transfer irreversibility, fluid friction irreversibility and Joule dissipation irreversibility) and it is given by

$$
B e=\frac{N y_{i}}{N s_{i}}=\frac{N y_{i}}{N y_{i}+N f_{i}+N m_{i}}
$$

This is employed to understand the entropy generation mechanisms as proposed by Bejan (1996, 1979). The value of $B e \rightarrow 1$ indicates that the heat transfer irreversibility dominates. On the other hand, $B e \rightarrow 0$ indicates that the irreversibility due to fluid friction and magnetic field dominates over the irreversibility due to the heat transfer. It is obvious that $B e=0.5$ is the case in which the irreversibility due to heat transfer is equal to sum of fluid friction and magnetic field contributions in the entropy generation.

\section{RESULTS AND DISCUSSION}

The closed form solutions for the flow of two immiscible micropolar fluids are obtained and reported in the previous section. These solutions are evaluated numerically and depicted graphically. The variations of velocity, micro-rotation, temperature, entropy generation number and Bejan number for different values of parameters are shown through figures.

\subsection{Flow field}

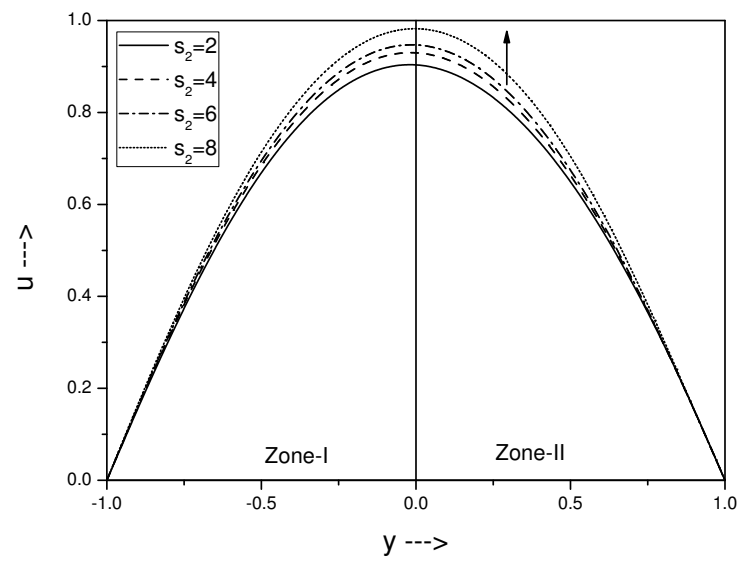

Fig. 2 Effect of $s_{2}$ on $u$ for $B=-1.5, c_{1}=0.6, c_{2}=0.7, H a=1$, $n_{\beta}=0.9, n_{\mu}=0.9, n_{\sigma}=0.9, R e=2, s_{1}=5$.

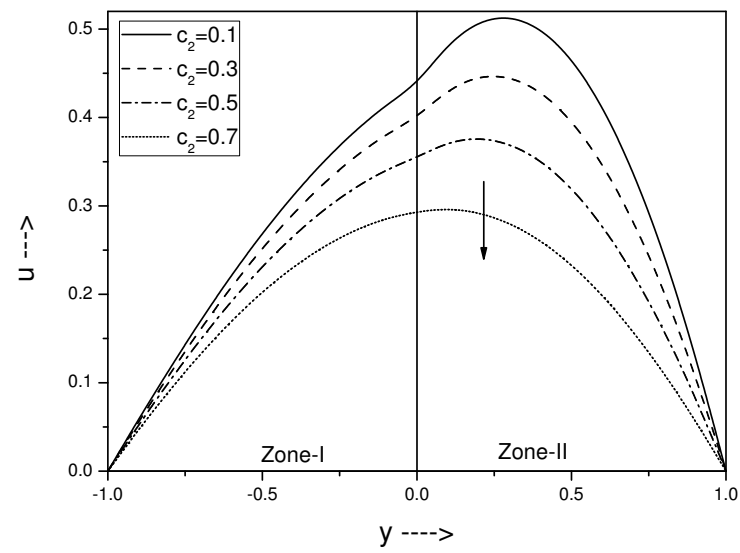

Fig. 3 Effect of $c_{2}$ on $u$ for $B=-1.5, c_{1}=0.8, H a=0.5, n_{\beta}=0.9$, $n_{\mu}=0.9, n_{\sigma}=0.5, R e=1.5, s_{1}=2, s_{2}=3$.

The effect of the couple stress parameter $s_{2}$ on the velocity field is shown in Fig. 2. It is seen that as $s_{2}$ increases, the velocity increases in both the zones of the channel. As $s_{2} \rightarrow \infty$, we get the case of Newtonian (viscous) fluids. The effect of the micropolarity parameter $c_{2}\left(0 \leq c_{2}<1\right)$ on the velocity field is shown in Fig. 3. It is seen that as $c_{2}$ increases, the velocity decreases in both zones of the channel. The velocity in case of micropolar fluid is less than that of the viscous fluid ( $c_{2} \rightarrow 0$ corresponds to viscous fluid). 


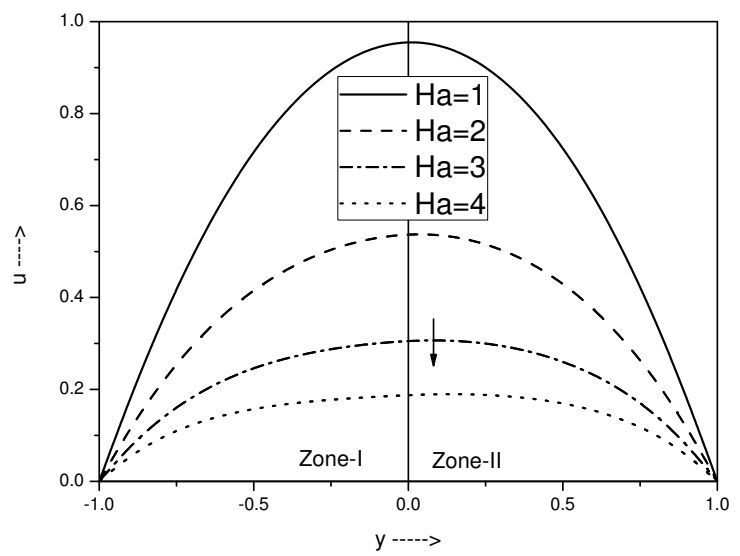

Fig. 4 Effect of $H a$ on $u$ for $B=-3, c_{1}=0.9, c_{2}=0.9, R e=4$, $n_{\beta}=0.8, n_{\mu}=0.9, n_{\sigma}=0.8, s_{1}=8, s_{2}=8$.

Fig. 4 shows the effect of the Hartmann number $\mathrm{Ha}$ (magnetic parameter) on the velocity field. As we expected, the velocity profiles are symmetrical about the centerline of the channel. It is clear that, increasing the value of $\mathrm{Ha}$ have a tendency to slow down the fluid motion. This is because of the presence of the transverse magnetic field, which creates a resistive force similar to the drag force that acts in the opposite direction of the fluid motion, thus causing the velocity of the fluid to decrease. This shows that the imposed magnetic field has a retarding influence on the flow field.

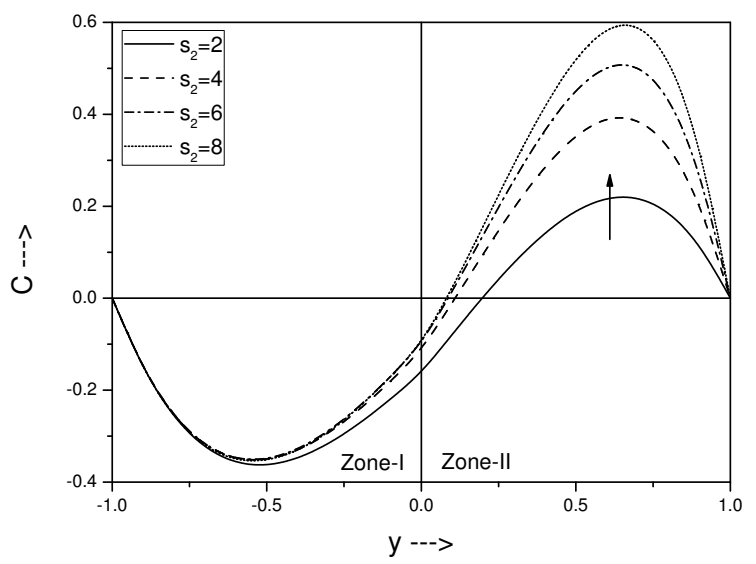

Fig. 5 Effect of $s_{2}$ on $\mathcal{C}$ for $B=-2, c_{1}=0.9, c_{2}=0.8, H a=0.5$, $n_{\beta}=0.8, n_{\mu}=0.8, n_{\sigma}=0.8, R e=6, s_{1}=5$.

The effect of the couple stress parameter $s_{2}$ is found to decrease the micro-rotational velocity drastically as seen in zone II and slightly in zone I as shown in Fig. 5. From Fig. 6, we observe that the micro-rotation changes sign from increasing nature to decreasing character in the zone II and in zone I the rotational velocity is increasing. The micro-rotation tends to zero as is expected that in the limit $\kappa \rightarrow 0$ i.e., $c_{2} \rightarrow 0$, the eqns. (1) and (2) are uncoupled with (3) and they reduce to viscous fluid flow equations. This may be due to increase in rotation of particles in zone II.

Fig. 7 shows the effect of the Hartmann number $\mathrm{Ha}$ on the microrotation field. As the Hartmann number $\mathrm{Ha}$ increases, micro-rotation changes its increasing nature to decreasing nature in zone II and as $\mathrm{Ha}$ increases, micro-rotation decreases numerically in the channel.

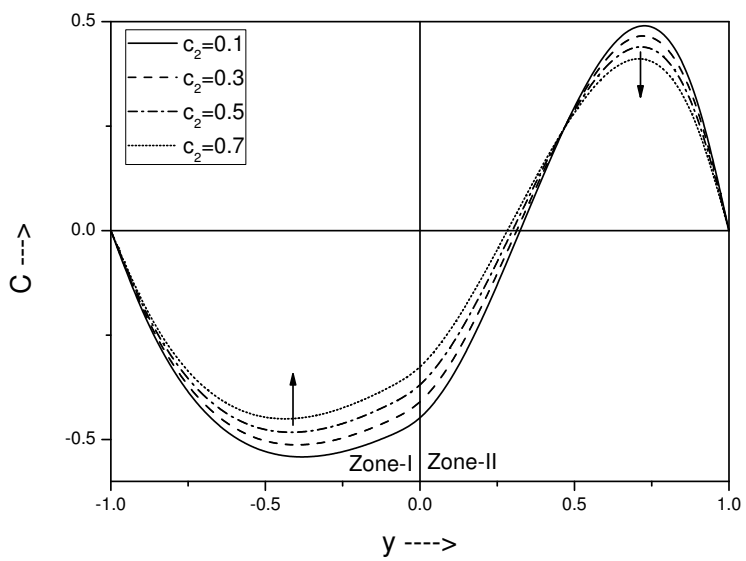

Fig. 6 Effect of $c_{2}$ on $\mathcal{C}$ for $B=-2, c_{1}=0.9, H a=1, n_{\beta}=0.6$, $n_{\mu}=0.6, n_{\sigma}=0.8, R e=6, s_{1}=4, s_{2}=4$.

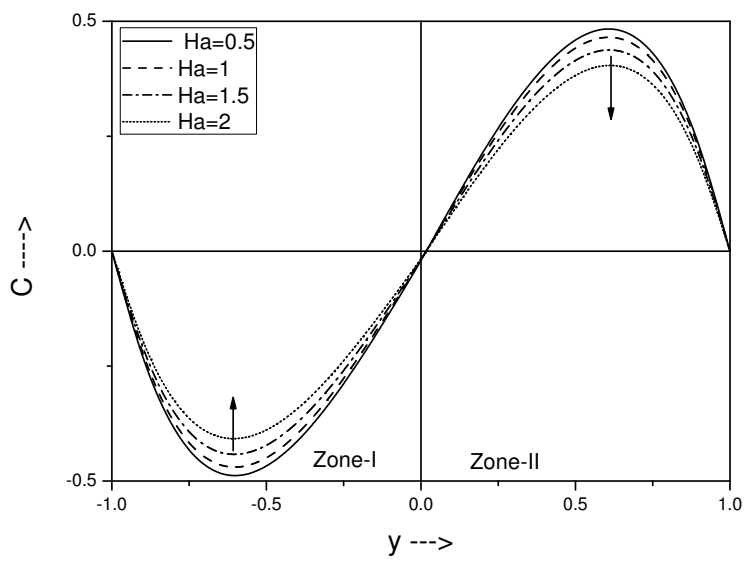

Fig. 7 Effect of $H a$ on $\mathcal{C}$ for $B=-4, c_{1}=0.9, c_{2}=0.9, n_{\beta}=0.9$, $n_{\mu}=0.9, n_{\sigma}=0.9, R e=6, s_{1}=5, s_{2}=5$.

\subsection{Thermal field and Heat transfer}

The effect of the couple stress parameter $s_{2}$ on temperature field is shown in Fig. 8. It is seen that as $s_{2}$ increases, the temperature increases in both zones of the channel. This may be due to the fact that velocity increases and hence dissipation increases (see fig 2). Fig. 9 depicts the effect of the micropolarity parameter $c_{2}$ on temperature field. It is observed that the temperature decreases with the increase of parameter $c_{2}$. Since velocity is decreasing, dissipation of energy due to velocity decreases and hence temperature decreases.

Fig. 10 displays the effect of the Hartmann number $\mathrm{Ha}$ on the temperature field. As the Hartmann number $\mathrm{Ha}$ increases, temperature decreases in both the regions of the channel. As Ha increases due to increasing magnetic field intensity, the fluid temperature decreases within the channel. This behavior is attributed to decrease the fluid velocity due to the magnetic field as shown in Fig. 4. Fig. 11 displays the effect of Brinkman number $\mathrm{Br}$ on temperature. It is observed that the temperature of both the fluids increases with increase in Brinkman number $\mathrm{Br}$. This may be due to viscous dissipation.

Fig. 12 shows that as Reynolds number Re increases, Nusselt number $N u$ increases. 


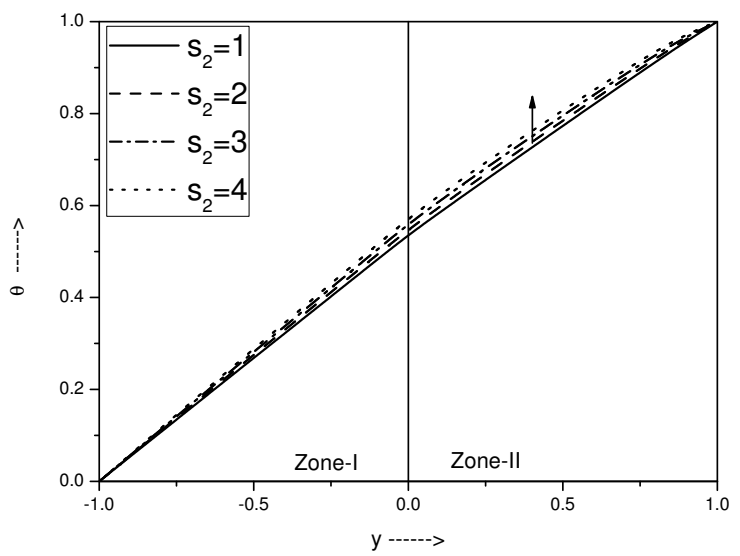

Fig. 8 Effect of $s_{2}$ on $\theta$ for $B=-1.5, B r=0.5, c_{1}=0.7, c_{2}=0.7$, $\delta_{1}=0.7, \mathrm{Ha}=0.5, n_{\beta}=0.9, n_{k}=1.1, n_{\mu}=0.6, n_{\sigma}=0.8$, $R e=2, s_{1}=6$.

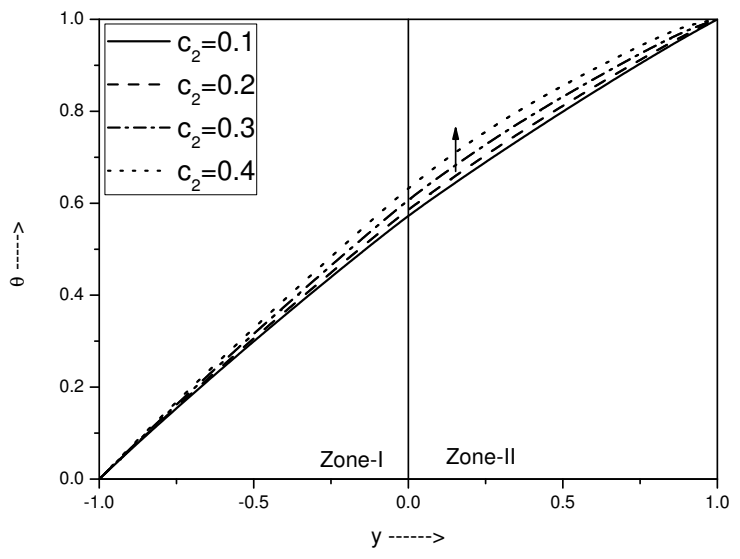

Fig. 9 Effect of $c_{2}$ on $\theta$ for $B=-1, B r=0.5, c_{1}=0.9, \delta_{1}=0.7$, $H a=0.5, n_{\beta}=0.9, n_{k}=1.1, n_{\mu}=0.5, n_{\sigma}=0.8, R e=0.5$, $s_{1}=6, s_{2}=6$.

\subsection{Entropy generation and Heat transfer irreversibility}

The transverse variation in heat transfer irreversibility in terms of entropy generation number $N s$ is shown in Figs. $13-16$ for different values of the parameters. Fig. 13 shows the effect of couple stress parameter $s_{2}$ on entropy generation number $N s$. As $s_{2}$ increases entropy generation increases drastically near the plates in zone II. Fig. 14 shows the effect of micropolarity parameter $c_{2}$ on entropy generation number $N s$. As $c_{2}$ increases entropy generation decreases in both the zones of the channel. The values of $N s$ when $c_{2}$ is near to zero are more than the values of $N s$ when $c_{2}$ is tending to 1 . Since the case $c_{2} \rightarrow 0$ represents viscous fluid case, the entropy generation rate in this special case is more than that of the micropolar fluid case.

Fig. 15 shows the variation of entropy generation number $N s$ as a function of $y$ at different Hartmann numbers $H a$. As $H a$ increases, $N s$ decreases drastically near the plates and has negligibly small effect at the interface. As Hartmann number increases, the curve for an entropy generation number becomes flat for a considerable distance in the middle of the channel.

The entropy generation number $N s$ is plotted in Fig. 16 as a func-

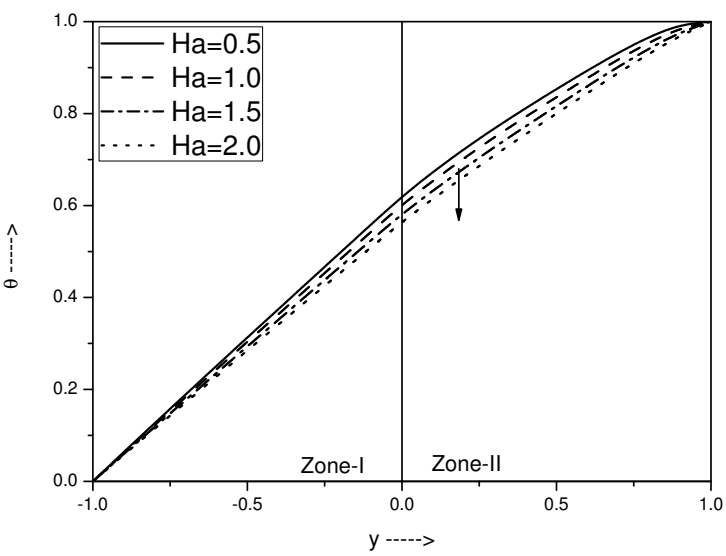

Fig. 10 Effect of $H a$ on $\theta$ for $B=-1.5, B r=0.5, c_{1}=0.9, c_{2}=0.9$, $\delta_{1}=0.7, n_{\beta}=0.9, n_{k}=1.1, n_{\mu}=0.6, n_{\sigma}=0.8, R e=2.5$, $s_{1}=8, s_{2}=8$.

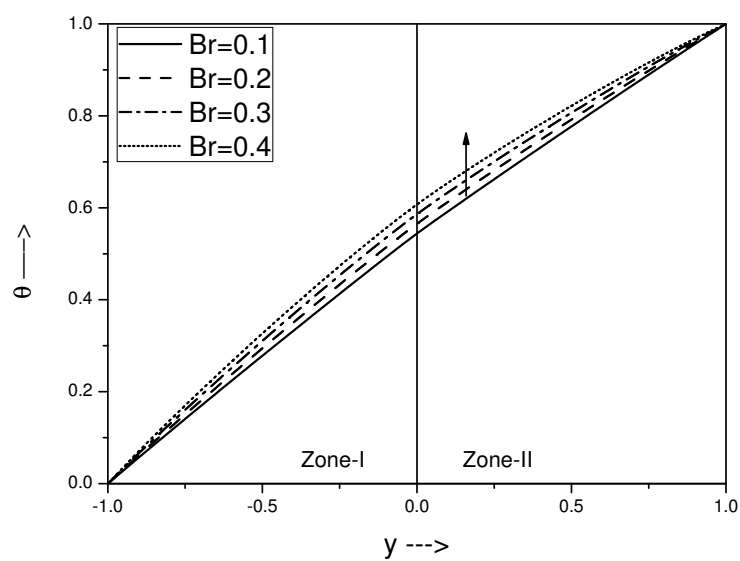

Fig. 11 Effect of $B r$ on $\theta$ for $B=-1.5, c_{1}=0.9, c_{2}=0.9, \delta_{1}=0.7$, $H a=3, n_{\beta}=0.9, n_{k}=1.1, n_{\mu}=0.6, n_{\rho}=0.8, n_{\sigma}=0.8$, $R e=1.5, s_{1}=8, s_{2}=8$.

tion of transverse direction y for different values of the viscous dissipation parameter $(B r / \Omega)$. This parameter is an important dimensionless number in the entropy generation analysis. It determines the relative importance of viscous effects to temperature gradient entropy generation. The entropy generation near the plates increases more rapidly in the zone I than in the zone II. The more the viscosity of the fluid is, the more the entropy generation. It is observed that since $N s$ takes the least value at the interface, the fluid friction irreversibility dominates at the interface of the channel. Again the entropy generation near the plates increases with increasing the viscous dissipation parameter $(B r / \Omega)$. For $(B r / \Omega)=0$, fluid friction irreversibility has no contribution to an entropy generator which falls to zero in the entire channel.

The transverse variation in heat transfer irreversibility in terms of Bejan number $B e$ is shown in Figs. 17 - 20 for different values of the parameters. Fig. 17 shows the effect of couple stress parameter $s_{2}$ on Bejan number $\mathrm{Be}$. As $s_{2}$ increases, Bejan number decreases. At the interface the variation in $\mathrm{Be}$ is rapid. Fig. 18 shows the effect of micropolarity parameter $c_{2}$ on Bejan number $B e$. As $c_{2}$ increases, Bejan number increases from 0.992 to 1 within a small range. Which implies $c_{2}$ almost 


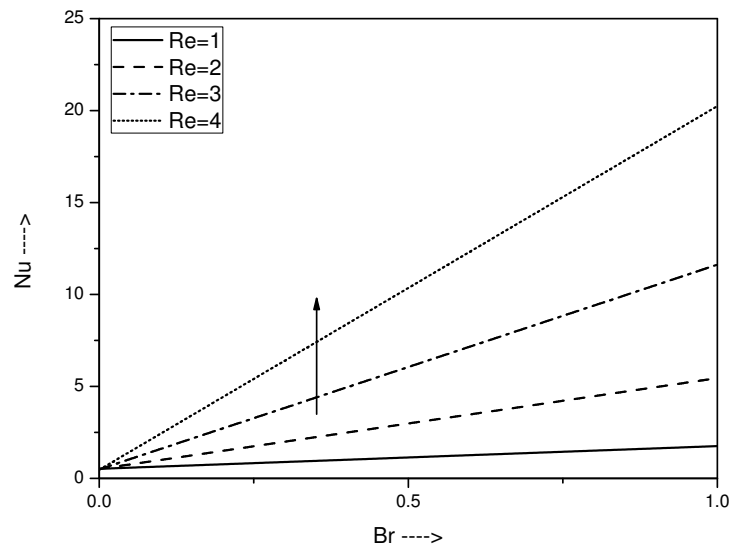

Fig. 12 Effect of $R e$ on Nusselt number $N u$ as a function of $\mathrm{Br}$ for $B=-2, c_{1}=0.2, c_{2}=0.3, H a=0.5, n_{\mu}=0.6, \delta_{1}=0.7$, $n_{\beta}=0.9, n_{k}=1.1, s_{1}=6, s_{2}=6$.

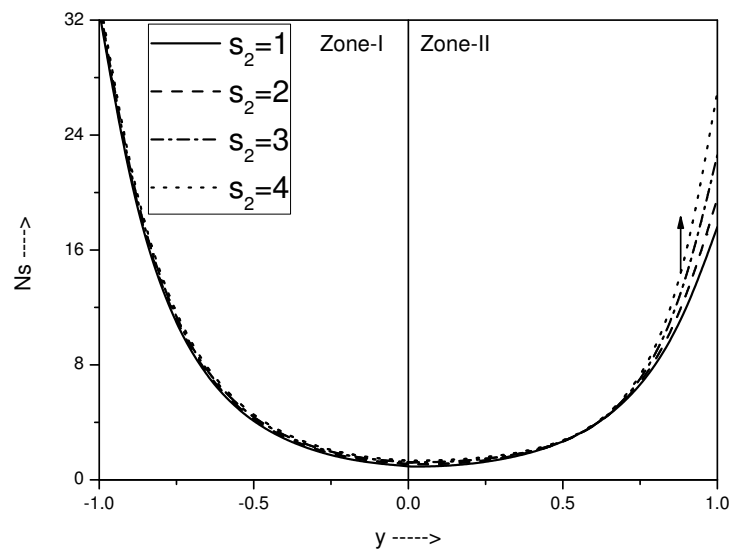

Fig. 13 Effect of $s_{2}$ on $N s$ for $B=-1, B r=0.1, c_{1}=0.3, c_{2}=0.4$, $\delta_{1}=0.9, H a=0.1, R e=1, n_{\beta}=0.9, n_{k}=1.1, n_{\mu}=0.9$, $n_{\sigma}=0.8, s_{1}=8, \Omega=1$.

has no effect on $B e$.

Fig 19 displays the variation of Bejan number $B e$ as a function of $\mathrm{y}$ at different Hartmann numbers $\mathrm{Ha}$. As $\mathrm{Ha}$ increases, $\mathrm{Be}$ increases drastically near the plates and has negligibly small effect at the interface. As Hartmann number increases, the curve for Bejan number becomes flat for a considerable distance in the middle of the channel. This shows that magnetic effect decreases friction at the walls but increases the entropy generation rate at the interface. This is a significant observation that can be made from the present study in view of its practical applicability.

The Bejan number $B e$ is shown in Fig. 20 as a function of transverse direction $y$ for different values of viscous dissipation parameter $(B r / \Omega)$. At $(B r / \Omega)=0$, the contribution of fluid friction irreversibility to overall entropy generation is absent, thus resulting in an invariant distribution of Bejan number. Bejan number shows its maximum value $B e=1$ which means there is no entropy generation due to fluid friction. The Bejan number is highest at the interface of the channel and decreases as we move towards the channel walls on either direction. The Bejan number decreases as the viscous dissipation parameter $(B r / \Omega)$ increases.

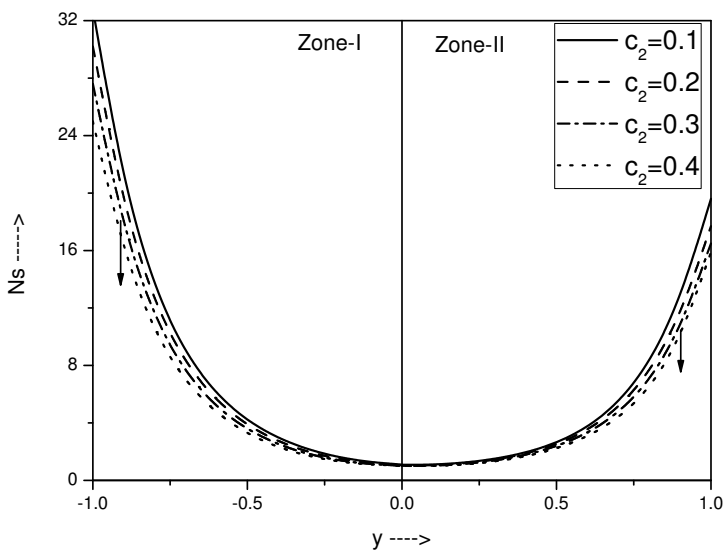

Fig. 14 Effect of $c_{2}$ on $N s$ for $B=-1, B r=0.3, c_{1}=0.3, \delta_{1}=0.9$, $H a=0.3, R e=2, n_{\beta}=0.9, n_{k}=1.1, n_{\mu}=0.9, n_{\sigma}=0.8$, $s_{1}=6, s_{2}=6, \Omega=1$.

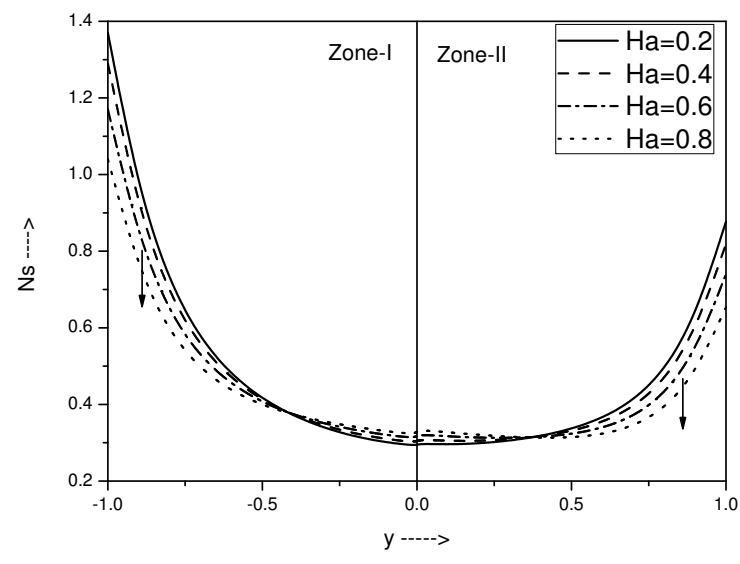

Fig. 15 Effect of $H a$ on $N s$ for $B=-0.1, B r=0.1, c_{1}=0.2$, $c_{2}=0.2, \delta_{1}=0.9, R e=5, n_{\beta}=0.9, n_{k}=1.1, n_{\mu}=0.9$, $n_{\sigma}=0.8, s_{1}=8, s_{2}=8, \Omega=1$.

\section{CONCLUSION}

The MHD flow of two immiscible incompressible micropolar fluids in a horizontal channel of width $2 h$ with the plates at constant temperature, under the influence of a constant pressure gradient has been analyzed in terms of entropy generation rate. It is observed that

1. The higher values of the micropolarity parameter $c_{2}$ (i.e., the effect of micro-rotation becomes significant) result in lowering the velocity distribution and the presence of couple stresses in the fluid decreases the velocity.

2. The values of $N s$ near the plates are more than they are at the interface, indicating that friction due to surface on the fluids increases entropy generation rate.

3. The values of $N s$ in zone-I are more than they are in the zone-II near the plates. This indicates that the more is the viscosity of the fluid, the more is the entropy generation rate.

4. Increasing Hartmann number $\mathrm{Ha}$ (Magnetic parameter) tends to increase the values of Bejan number near the plates. The entropy generation number decreases near the plates with an increase in magnetic parameter and it attains almost flat shape in the middle of the channel. This implies 


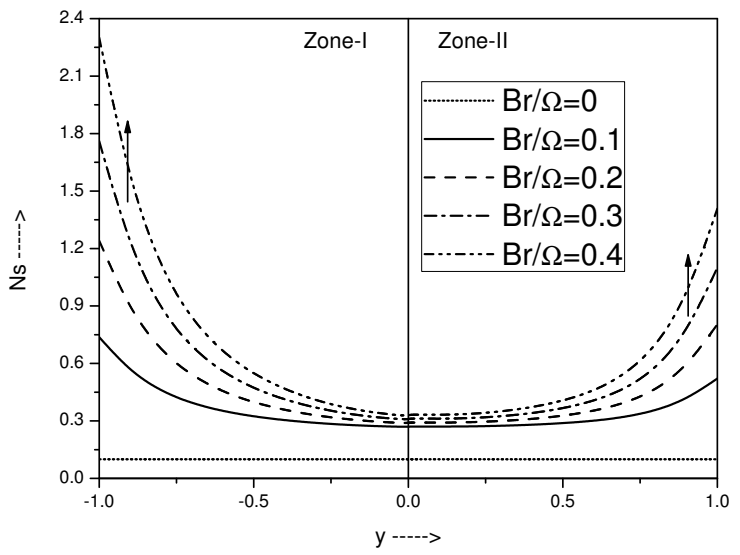

Fig. 16 Effect of $(B r / \Omega)$ on $N s$ for $B=-2, c_{1}=0.1, c_{2}=0.1$, $\delta_{1}=0.9, H a=0.2, R e=2, n_{\beta}=0.9, n_{k}=1.1, n_{\mu}=0.9$, $n_{\sigma}=0.8, s_{1}=8, s_{2}=8$.

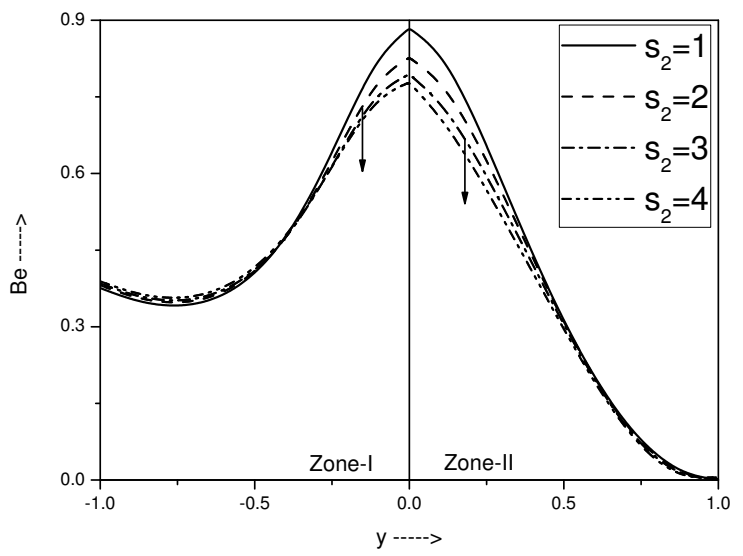

Fig. 17 Effect of $s_{2}$ on $B e$ for $B=-0.1, B r=0.1, c_{1}=0.2, c_{2}=0.2$, $\delta_{1}=0.9, H a=0.1, R e=10, n_{\beta}=0.9, n_{k}=1.1, n_{\mu}=0.9$, $n_{\sigma}=1.1, s_{1}=8, \Omega=1$.

that magnetic field reduces the fluid friction near the walls.

5. The walls act as strong sources of irreversibility since the viscous shear stress on the walls is maximum due to momentum transfer from the plate to the fluid. This shows an industrial application that micropolar fluids with high micropolarity and less couples stresses will act as good lubricants.

\section{ACKNOWLEDGEMENTS}

The authors thank the anonymous reviewers for their valuable comments which led to the improvement of the paper.

\section{NOMENCLATURE}

$\begin{array}{ll}B e & \text { Bejan number }\left(=\frac{1}{1+\phi}\right) \\ B r & \text { Brinkman number }(=E k \text { Pr }) \\ \frac{B r}{\Omega} & \text { viscous dissipation parameter } \\ c_{1}, c_{2} & \text { micropolarity or coupling numbers } \\ \mathcal{C} & \text { non-dimensional micro-rotation in z-direction } \\ d_{i j} & \text { components of the strain }\end{array}$

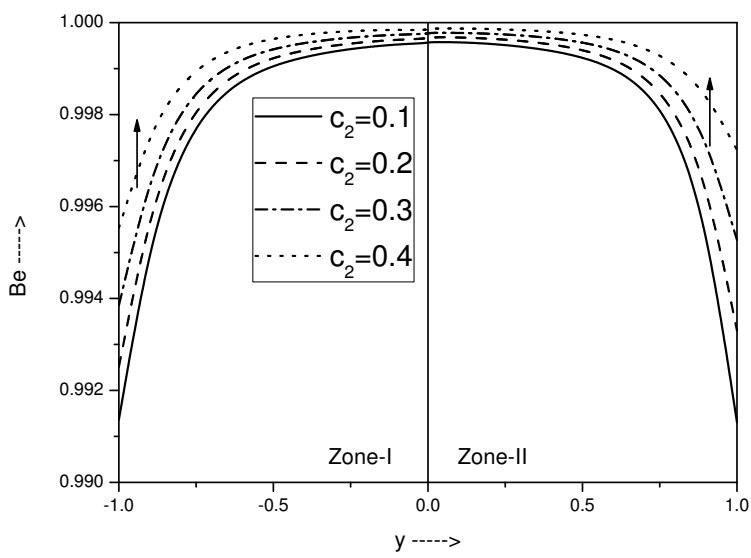

Fig. 18 Effect of $c_{2}$ on $B e$ for $B=-0.3, B r=0.2, c_{1}=0.2, \delta_{1}=0.9$, $H a=0.2, R e=6, n_{\beta}=0.9, n_{k}=1.1, n_{\mu}=0.9, n_{\sigma}=1.1$, $s_{1}=6, s_{2}=6, \Omega=1$.

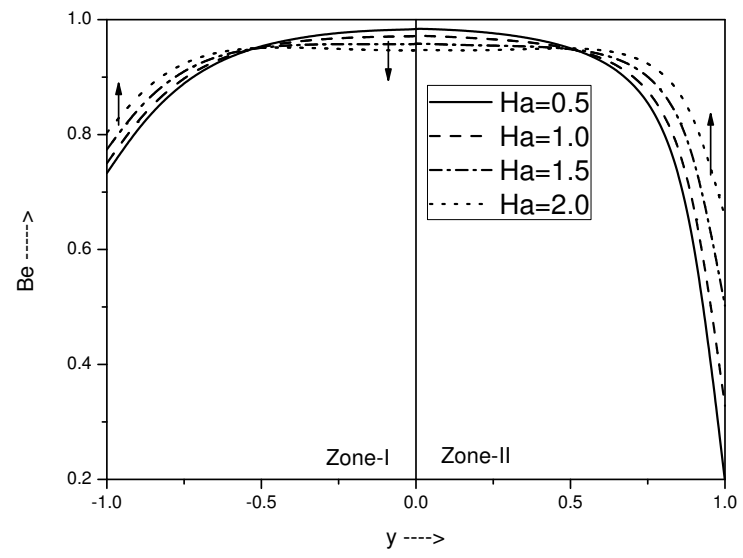

Fig. 19 Effect of $H a$ on $B e$ for $B=-0.1, B r=0.1, c_{1}=0.5$, $c_{2}=0.5, \delta_{1}=0.9, R e=2, n_{\beta}=0.9, n_{k}=1, n_{\mu}=0.9$, $n_{\sigma}=1.1, s_{1}=8, s_{2}=8, \Omega=1$.

$D \quad$ deformation tensor

$E \quad$ specific internal energy

$\vec{E} \quad$ electric field

Ek Eckert number

$\vec{f} \quad$ body forces per unit mass

$h_{o} \quad$ induced magnetic field

$\overrightarrow{\boldsymbol{H}} \quad$ total magnetic field

$H_{o} \quad$ applied magnetic field

$\mathrm{Ha}$ Hartmann number

2h height of the free channel

$\overrightarrow{\mathbf{h}} \quad$ heat flux

$\bar{J} \quad$ current density

$j \quad$ gyration coefficient

$k_{1}, k_{2} \quad$ thermal conductivity of the fluid in zone-I,II

$\vec{\ell} \quad$ body couple per unit mass

$m_{i j} \quad$ couple stress tensor

$N f_{i} \quad$ entropy generation due to viscous dissipation

$N s_{i} \quad$ dimensionless total entropy generation number

$N y_{i} \quad$ entropy generation due to transverse conduction 


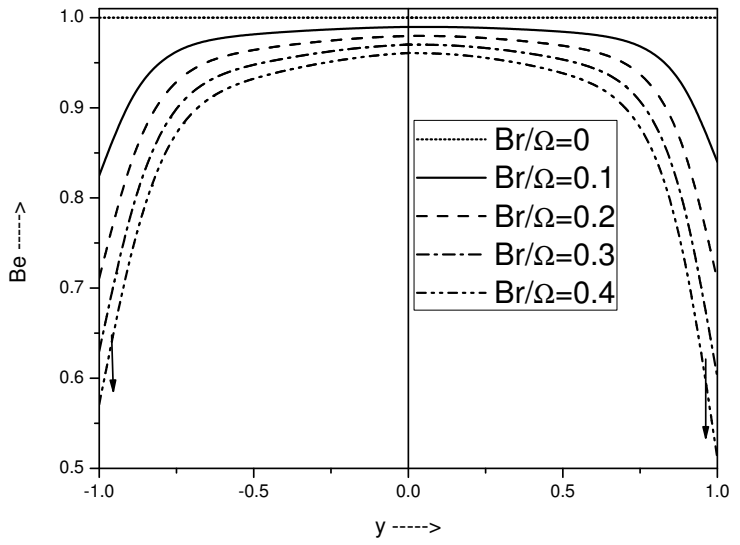

Fig. 20 Effect of $(B r / \Omega)$ on $B e$ for $B=-0.5, c_{1}=0.5, c_{2}=0.5$, $\delta_{1}=0.9, H a=0.1, R e=10, n_{\beta}=0.9, n_{k}=1.1, n_{\mu}=0.9$, $n_{\sigma}=1.1, s_{1}=10, s_{2}=10$.

\section{$\mathrm{Nu} \quad$ Nusselt number}

$n_{\beta} \quad$ ratio of couple stress viscosity coefficients $\left(=\frac{\beta_{2}}{\beta_{1}}\right)$

$n_{k} \quad$ ratio of thermal conductivities $\left(=\frac{k_{2}}{k_{1}}\right)$

$n_{\mu} \quad$ ratio of viscosities $\left(=\frac{\mu_{2}}{\mu_{1}}\right)$

$n_{\rho} \quad$ ratio of densities $\left(=\frac{\rho_{2}}{\rho_{1}}\right)$

$n_{\sigma} \quad$ ratio of electric conductivities $\left(=\frac{\sigma_{2}}{\sigma_{1}}\right)$

$p \quad$ pressure

$\operatorname{Pr} \quad$ Prandtl number

$\vec{q} \quad$ velocity vector

$q_{1}, q_{2} \quad$ non-dimensional volumetric flow rates in zones

Re Reynolds number

$s_{1}, s_{2} \quad$ couple stress parameters in zone I,II

$\left(S_{i}\right)_{G} \quad$ entropy generation rate

$\left(S_{i}\right)_{G, C} \quad$ characteristic entropy transfer rate

$t_{i j} \quad$ stress tensor

$T_{1}, T_{2} \quad$ non-dimensional temperatures of the plates

$u \quad$ non-dimensional velocity in $\mathrm{X}$-direction

\section{GREEK SYMBOLS}

$\alpha, \beta, \gamma \quad$ gyration viscosity coefficients

$\delta_{i j} \quad$ kronecker delta

$\delta_{1} \quad$ couplestress parameter $\left(=\frac{\beta_{1}}{\mu_{1} h^{2}}\right)$

$\epsilon_{i j k} \quad$ Levi-Civita symbol or permutation symbol

$\vec{\nu} \quad$ micro-rotation vector

$\Omega \quad$ dimensionless temperature difference $\left(=\frac{\Delta T}{T_{o}}\right)$

$\Phi \quad$ dissipation function

$\phi \quad$ irreversibility ratio $\left(=\frac{N f}{N y}\right)$

$\rho \quad$ density

$\theta \quad$ non-dimensional temperature

\section{REFERENCES}

Alpher, R.A., 1961, "Heat transfer in magnetohydrodynamic flow between parallel plates," Int J Heat Mass Transf, 3(2), 108-112.

Ariman, T., and Cakmak, A.S., 1968, "Some basic viscous flows in micropolar fluids," Rheol Acta, 7(3), 236-242.

http://dx.doi.org/10.1007/bf01985784.

Ariman, T., Turk, M.A., and Sylvester, N.D., 1973, "Application of microcontinuum fluid mechanics," Int J Eng Sci, 12(4), 273-293.
Bakhtiyarov, S.I., and Siginer, D.A., 1997, "A note on the laminar coreannular flow of two immiscible fluids in a horizontal tube," Symposium on liquid-liquid two phase flow and transport phenomena, Begell house, Inc. Santa Barbara, 107-111.

Bejan, A., 1979, "A study of entropy generation in fundamental convective heat transfer," ASME J Heat Transf, 101(4), 718-725.

http://dx.doi.org/10.1115/1.3451063.

Bejan, A., 1980, "Second law analysis in heat transfer," Energy, 5(8-9), 720-732.

Bejan, A., 1982, "Second law analysis in heat transfer and thermal design," Adv Heat Transf, 15, 1-58.

Bejan, A., 1996, Entropy Generation Minimization, CRC Press, Boca Raton, New York.

Bhattacharya, R.N., 1968, "The flow of immiscible fluids between rigid plates with a time dependent pressure gradient," Bull Calcutta Math Soc, 1, 129-137.

Bhattacharyya, K., Mukhopadhyay, S., Layek, G.C., and Pop, I., 2012, "Effects of thermal radiation on micropolar fluid flow and heat transfer over a porous shrinking sheet," Int J Heat Mass Transf, 55(1), 29452952.

http://dx.doi.org/10.1016/j.ijheatmasstransfer.2012.01.051.

Bird, R.B., Stewart, W.E., and Lightfoot, E.N., 1960, Transport Phenomena, John Wiley \& Sons, New York.

Chamkha, A.J., 1998, "Mixed convection flow along a vertical permeable plate embedded in a porous medium in the presence of a transverse magnetic field," Numer Heat Transf, 34(1), 93-103.

http://dx.doi.org/10.1080/10407789808913979.

Chamkha, A.J., 2000, "Flow of two immiscible fluids in porous and nonporous channels," J Fluids Eng, 122(1), 117-124.

http://dx.doi.org/10.1115/1.483233.

Chamkha, A.J., and Quadri, M.A., 2001, "Heat and mass transfer from a permeable cylinder in a porous medium with magnetic field, and heat generation, absorption effect," Numer Heat Transf, 40(4), 387-401.

http://dx.doi.org/10.1080/104077801753238167.

Chaturani, P., and Samy, R.P., 1985, "A study of non-Newtonian aspects of blood flow through stenosed arteries and its applications in arterial diseases," Biorheol, 22(6), 521-531.

Damseh, R.A., Al-Odat, M.Q., and Al-Nimr, M.A., 2008, "Entropy generation during fluid flow in a channel under the effect of transverse magnetic field," Heat Mass Transf, 44(8), 897-904.

http://dx.doi.org/10.1007/s00231-007-0342-8.

Eringen, A.C., 1960, Microcontinuum field theories: II, Fluent media, Springer, New York.

Eringen, A.C., 1966, "The theory of micropolar fluids," J Math Mech, 16, $1-18$.

Jena, S.K., and Mathur, M.N., 1981, "Similarity solutions for laminar free convection flow of a thermo-micropolar fluid past a non-isothermal vertical flat plate," Int J Eng Sci, 19(11), 1431-1439.

Kamisli, F., and Hakan, F.O., 2008, "Second law analysis of the 2D laminar flow of two immiscible, incompressible viscous fluids in a channel," Numer Heat Transf, 44(6), 751-761.

http://dx.doi.org/10.1007/s00231-007-0308-x.

Kapur, J.N., 1964, "The flow of incompressible immiscible fluids between two parallel plates," Appl Sci Res, 13, 55-60.

Kiyasatfar, M., Pourmahmoud, N., Golzan, M.M., and Mirzaee, I., 2012, "Thermal behavior and entropy generation rate analysis of a viscous flow in MHD micropumps," J Mech Sci Technol, 26(6), 1949-1955.

http://dx.doi.org/10.1007/s12206-012-0401-z. 
Komurgoz, G., Arikoglu, A., and Ozkol, I., 2012, "Analysis of the magnetic effect on entropy generation in an inclined channel partially filled with a porous medium," Numer Heat Transf, 61(10), 786-799.

http://dx.doi.org/10.1080/10407782.2012.672890.

Lukaszewicz, G., 1999, Micropolar fluids: Theory and applications, Birkhauser, Springer, Boston.

Mahian, O., Mahmud, S., and Pop, I., 2012, "Analysis of first, second laws of thermodynamics between two isothermal cylinders with relative rotation in the presence of MHD flow," Int J Heat Mass Transf, 55(1718), 4808-4816.

http://dx.doi.org/10.1016/j.ijheatmasstransfer.2012.04.048.

Malashetty, M.S., Umavathi, J.C., and Prathap Kumar, J., 2006, "Magnetoconvection of two immiscible fluids in vertical enclosure," Heat Mass Transf, 42(11), 977-993.

http://dx.doi.org/10.1007/s00231-005-0062-x.

Muthuraj, R., and Srinivas, S., 2010, "Fully developed MHD flow of a micropolar and viscous fluids in a vertical porous space using HAM," Int J Appl Math Mech, 6(11), 55-78.

Nikodijevic, D., Milenkovic, D., and Stamenkovic, Z., 2011, "MHD Couette two fluid flow and heat transfer in presence of uniform inclined magnetic field," Heat Mass Transf, 47(12), 1525-1535.

http://dx.doi.org/10.1007/s00231-011-0815-7.
Paoletti, S., Rispoli, F., and Sciubba, E., 1989, "Calculation exergetic loses in compact heat exchanger passages," ASME AES, Int J Heat Mass Transf, 10(2), 21-29.

Prathap Kumar, J., Umavathi, J.C., Chamkha, A.J., and Pop, I., 2011, "Fully developed free convective flow of micropolar and viscous fluids in a vertical channel," Appl Math Model, 34(5), 1175-1186. http://dx.doi.org/10.1016/j.apm.2009.08.007.

Rao, A.R., and Usha, S., 1979, "Peristaltic transport of two immiscible viscous fluids in a circular tube," J Fluid Mech, 298, 271-285. http://dx.doi.org/10.1017/S0022112095003302.

Rees, D.A.S., and Bossom, A.P., 1996, “The Blasius boundary layer flow of a micropolar fluid," Int J Eng Sci, 34(1), 113-124.

Sandeep, P., and Deshpande, M.D., 2003, A note on the no-slip boundary condition, Technical Report, National Aerospace Laboratories, Bangalore, India.

Stokes, V.K., 1984, Theories of Fluids with Microstructures, Springer, New York.

Umavathi, J.C., Chamkha, A.J., Mateen, A., and Al-Mudhaf, A., 2005, "Unsteady two fluid flow and heat transfer in a horizontal channel," Heat Mass Transf, 42(2), 81-90.

http://dx.doi.org/10.1007/s00231-004-0565-x. 